\title{
ANÁLISIS LINGÜÍSTICO-SEMIÓTICO. LA VALORIZACIÓN UTÓPICA EN EL SPOT PUBLICITARIO
}

\section{LINGUISTIC-SEMIOTIC ANALYSIS. UTOPIC \\ VALUE IN BRAND SPOT}

\section{Lidia PELLICER GARCÍA}

\author{
Universidad de Murcia \\ lylipellica@hotmail.com
}

Resumen: La creación de la personalidad de la marca atiende a unos valores semántico-discursivos, siendo uno de ellos el valor utópico mediante el cual el sujeto realiza su propia identidad conjugándose con su objeto de valor.

Abstract: Branding personality creation has a semantics-discursives values. One of them is the utopic value with a subject does his own identity with his value object.

Palabras clave: Marca. Personalidad. Valor. Utópico.

Key Words: Branding. Personality. Value. Utopic.

\section{INTRODUCCIÓN}

Como señala Klein (2005: 36), en la actualidad es importante estudiar el valor de la marca, pues si bien las empresas pueden fabricar productos, lo 
que los consumidores compran son marcas (Tauber, 1988; Blackett, 1989; Lusch y Harvey, 1994).

Su estudio puede realizarse desde diferentes perspectivas: financiera, psicológica, económica, etc. (del Río Lanza, Vázquez Casielles e Iglesias Argüelles, 2002: 87). No obstante, en el presente artículo recurriremos a un análisis semiótico, siguiendo los postulados de Semprini (1993: 25), quien afirma que la marca es una máquina que produce y atribuye significación en aras de construir su propia personalidad.

La creación de dicha personalidad de marca atiende a la creación de unos valores que van a ser estudiados teniendo en cuenta a Floch (1990: 176). De todos los valores de marca que posteriormente se presentarán, el presente artículo se centrará en el valor utópico que será aplicado al spot de televisión realizado con motivo de la campaña de lanzamiento de Movistar, de Telefónica, en 1996.

\section{VALORIZACIONES DE MARCA}

Floch (1990: 176) analiza la comunicación de Citroën de los años 80 sirviéndose de cuatro conceptos dispuestos alrededor de una estructura gráfica de forma rectangular; se trata del cuadrado semiótico de la axiología del consumo:

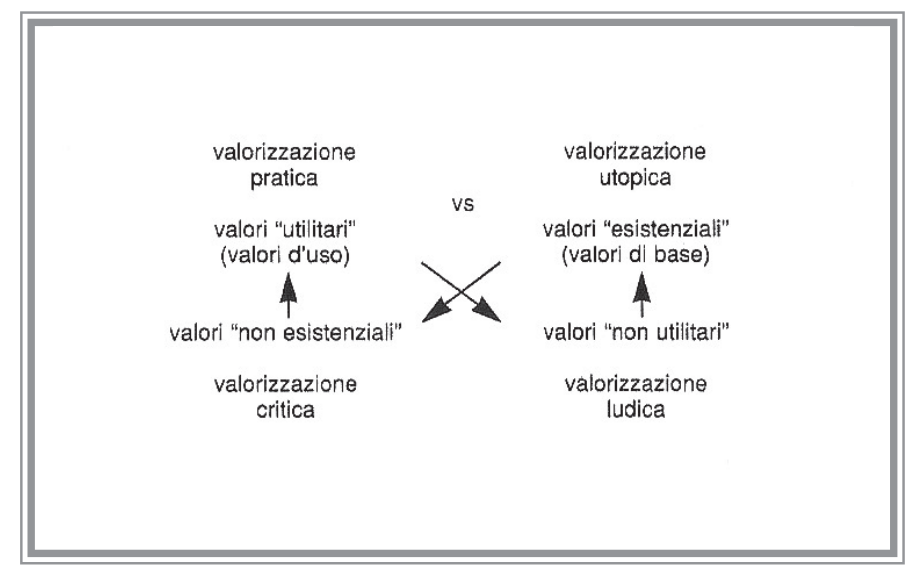

Tabla 15. Cuadrado semiótico de la axiología del consumo:

Valorizaciones (Floch, 1990: 176) 
A este respecto, Semprini destaca que estos cuatro conceptos son obtenidos a partir de la oposición semionarrativa, elaborada originariamente por Greimas (1966), del valor de base vs. valor de uso. El primero se caracteriza por ser un valor profundo, potente y universal capaz de justificar la puesta en marcha del motor de la narración y la acción del sujeto. En relación a ello, Semprini señala lo siguiente:

Tali valori sono definiti di base non solamente perché più profondi o universali, ma anche perché essi determinano la tensione fondamentale del racconto, nella misura in cui la loro acquisizione (o non acquisizione) costituisce l obiettivo di fondo, il fine ultimo del soggetto (Semprini, 1993: 120).

Frente a este valor, el de uso se caracteriza por su carácter instrumental y limitado.

Da un lato essi intervengono, lungo tutta la narrazione, per giustificare ed orientare le azioni e i comportamenti del soggetto. D altro lato essi non trovano posto nella narrazione che in relazione ai valori di base, all acquisizione dei quali essi contribuiscono (Semprini, 1993: 120).

A partir de esta oposición, Floch redefine el valor de uso como valor práctico y el de base como valor utópico; valores que, junto con los otros dos, el crítico y el lúdico, Marrone (2002: 17) define de la siguiente manera:

- Valorización práctica. «Una valorizzazione pratica, con la quale si considera 1 oggetto soprattutto per il suo carattere di strumento».

- Valorización utópica. «Una valorizzazione utopica, mediante la quale il soggetto realizza la propia identità congiungendosi con il suo oggetto di valore».

- Valorización lúdico-estética. «Una valorizzazione ludico-estetica corrispondente alla negazione di quella pratica, dove si considera 1 oggetto, prescindendo dalla sua utilizzabilità, a partire dalle sue qualità formali e fisiche, dal piacere che procura, dalla sua belleza».

- Valorización crítica. Una valorización en la que «l oggetto viene scelto più che altro per la sua convenienza».

Tales valorizaciones, señala Marrone (1999:17), se corresponden con cuatro tipos de pasiones:

— La pasión de la necesidad-valorización práctica.

- La pasión del deseo-valorización utópica. 
— La pasión del placer-valorización lúdica.

— La pasión del interés-valorización crítica.

\section{EL VALOR UTÓPICO EN EL DISCURSO DE MOVISTAR}

Presentadas las diferentes valorizaciones, cabe preguntarse ¿cómo se crea semióticamente este valor en el discurso de Movistar? ¿Qué tipo de pasión caracteriza la personalidad de este producto-signo? Para resolver estas cuestiones, hemos seleccionado dos spots de televisión realizados con motivo de la campaña de lanzamiento de Movistar, de Telefónica, en 1996 y $1997^{1}$ (Pérez Yuste, 2002: 26-31), en los que analizaremos las estrategias semiótico-lingüísticas de creación del valor utópico en el discurso, teniendo en cuenta, como expresa Marrone (1999: 17), que la valorización utópica es aquella mediante la cual el sujeto realiza su propia identidad conjugándose con su objeto de valor.

\subsection{Valorización utópica, unión del sujeto con su objeto de valor. La autorrealización}

\subsubsection{El discurso de la existencia. El viajero profesional}

Nos encontramos con unos sujetos que van a experimentar en Movistar la posibilidad de fusionarse para obtener el uso modal del «poder-ser» con el que

\footnotetext{
${ }^{1}$ Pérez Yuste realiza un estudio sobre el proceso de la implantación de la telefonía móvil en España y señala al respecto de estos años que vamos a estudiar en este artículo que terminadas las Elecciones Generales de marzo de 1996, comienza un trienio de actuaciones conducentes a la plena liberalización de las infraestructuras y de los servicios de telecomunicación eligiéndose (por parte del Gobierno Popular) el "duopolio" basado en dos empresas públicas: Telefónica y Retevisión, su posterior privatización y, finalmente, la apertura total del mercado el 1 de diciembre de 1998.

Tras cambiar el Gobierno, se creó un segundo operador global de telecomunicaciones, el 7 de junio de 1996, otorgando a la empresa pública Retevisión un título habilitado para el servicio de telefonía básica así como para el servicio portador.

En ese mismo año se creó también la Comisión del Mercado de las Telecomunicaciones, como órgano gubernamental independiente, encargado de velar por las condiciones de competencia efectiva en el mercado, por la correcta formación de los precios y ejercer de órgano arbitral en los conflictos que surgiesen en el sector.

Una vez establecido el "duopolio" el siguiente paso fue iniciar el proceso de adjudicación de activos de ambas compañías. De esta forma, el 18 de enero de 1997 se puso a la venta el 20,9 \% de Telefónica, que estaba en manos del Estado, mediante la Oferta Pública de Venta (OPV) más publicitada de todos los tiempos (en 1994 Telefónica ya se había privatizado en un 12\%), dejando de ser una compañía pública en el plazo de un mes, igual que le sucedería a Retevisión el 11 de julio de 1997 pasando al consorcio formado por el operador italiano STET (Telecom Italia) y las Eléctricas Endesa y Unión FENOSA.
} 
realizarán su propia identidad. La apertura que caracteriza esta nueva etapa otorgada por el nuevo signo, por Movistar (Pellicer García, 2010: 149-166)², la hallamos también en la tierra donde el actante sujeto, como señala Marrone, se actorializa en un yo «rompedor de fronteras» gracias a la unión con Movistar:

l attante Soggetto si attorializza come un Io che svolge il programma di rompere barriere, attraversare confini, percorrere spazi infiniti, per realizzare il quale deve congiungersi con il telefonino, grazie a cui acquisisce la modalità del poter-fare (Marrone, 1999: 153).

¿Qué características semánticas se desprenden de esa conjunción sujetoobjeto de valor en la adquisición de la identidad del propio sujeto? ¿Cómo se figurativizan dichos contenidos en discurso?

Antes de responder a estas cuestiones vamos a detenernos en el sujeto que va a adquirir su identidad a través de la unión con su objeto de valor. El discurso nos muestra a dos tipos de sujetos: profesionales famosos y personajes anónimos. Todos ellos se caracterizan por desarrollar su acción en contacto con el objeto de valor en un mismo espacio: la ciudad. Nos encontramos, pues, con ciudadanos realizando su trabajo en la ciudad en contacto permanente con el objeto de valor.

Floch (1990: 71-73) nos habla del «viajero profesional», el cual está en relación con la «secuencia», «la fluidez», «la tensión», con el trayecto:

Los Profesionales de la construcción o la búsqueda de «secuencias». La secuencia es un término de los deportes, de la gimnasia o de la escalada en roca; lo elegimos porque ilustra en la fluidez, el poder y el dominio de técnicas que caracterizan a la verdad y a las hazañas de los Profesionales (Floch, 1990: 72).

Los viajeros, sean famosos o anónimos, realizan un trayecto, el cual cobra una significación que contribuye a crear el significado del objeto de valor. ¿Dónde realizan ese trayecto? En la ciudad, siendo, consecuentemente, el espacio urbano otra de las cualidades significativas en el discurso de la marca en estos años.

\footnotetext{
${ }^{2}$ Anteriormente a Movistar, la compañía de Telecomunicaciones de Telefónica contaba con el operador Moviline (1993-1998) presentado como un producto práctico, necesario y útil para la sociedad coetánea sin representación sígnica. Movistar surge como consecuencia de una competencia laboral mercantil, siendo imprescindible el paso de una materialidad práctica de un producto a una identificación espiritual de un nuevo signo-marca que garantice la personalidad de la empresa. Para ello Movistar es identificado mediante el imaginario de una estrella, adquiriendo el significado de apertura.
} 


\subsubsection{La representación del espacio urbano. Cine y publicidad}

El discurso de marca nos introduce en la representación del espacio urbano como espejo de la ciudad y en particular de la metrópoli contemporánea. La unión del sujeto y del objeto de valor se figurativiza en la urbanidad como emblema de la contemporaneidad, la cual es representada, como posteriormente veremos, a través de la creación de una estética de la vida cotidiana.

En este sentido Basso señala la relación entre cine y publicidad como muestra mundial de la realización hacia el futuro:

L occhio emotivo del cinema non poteva che filtrare la realtà urbana, porsi addirittura come un attore protagonista di ciò che filmava, non limitandosi quindi mai a fungere da mero «ruolo di spalla» rispetto alla documentazione della nuova, esaltante architettura metropolitana. Se come metteva giá in evidenza Vachel Lindsay 1915, il cinema si proponeva come una mostra mondiale permanente e continuamente itinerante delle idee e delle realizzazioni piú avanzate (tecnologicamente e esteticamente) in campo architettonico, era altrettanto evidente che la diagnosi sociale che i film contenevano, non trascurando di addentrarsi nelle periferie degradate e nei sobborghi, non era sempre favorevole all edificazione della nuova città e allo stile di vita che il progresso imponeva (Basso, 2003: 228-229).

Efectivamente, nos encontramos ante un espacio utópico estético en el que lo tecnológico vertebra el ambiente idealizándolo hacia el progreso y ocultando el espacio de la miseria y los suburbios sociales.

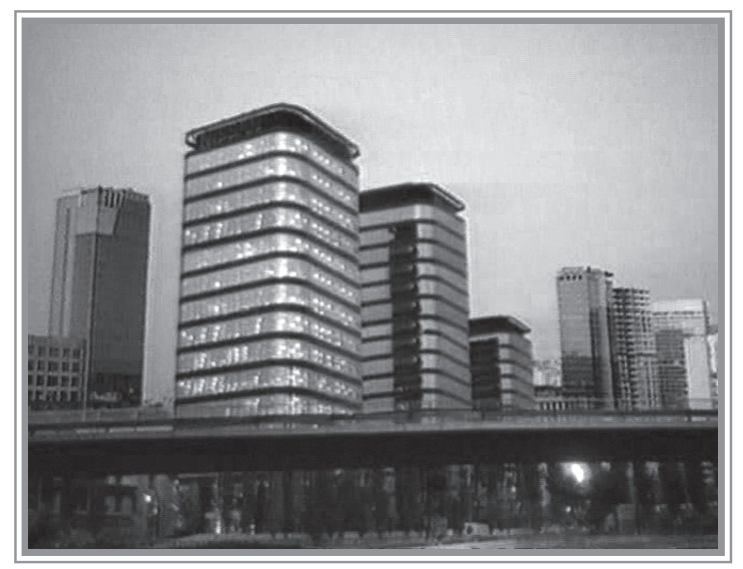

Fig.1. Imagen espacial de la ciudad, Movistar (Telefónica, 1996) 
En dicho espacio idealizado la posesión del objeto supone la pertenencia tecnológica para el poseedor, de hecho, aunque la pertenencia del objeto se enuncie y anuncie como posible para todo tipo de gente, es cierto que en esta etapa de la vida del signo se muestran sujetos de clase media-alta, como más adelante comentaremos.

Con todo, el sujeto del discurso portador del objeto de valor atraviesa la ciudad como un flâneur modello, como un perfecto paseante urbano, como señala Basso:

Il personaggio filmico che attraversa la città può divenire una sorta di flâneur modello, ossia un fruitore degli spazi metropolitani che segue perfettamente le prescrizioni implicate nei testi architettonici e nell organizzazione urbanistica (Basso, 2003: 230).

Un perfecto paseante urbano que adquirirá su identidad gracias a la conjunción con el objeto de valor en el espacio urbano. Veamos las características significativas que dicho objeto de valor adquiere en el proceso urbano.

En primer lugar, nos encontramos con unos viajeros que controlan, que tienen una maestría y unos conocimientos técnicos, una eficiencia del control. Este rasgo de identidad por el que es reconocido el viajero es resultado de la conjunción con el objeto de valor, la cual se figurativiza en la posesión del objeto de tal forma que podemos hablar de «práctica encarnada», como señala Semprini:

si tratta di una pratica incarnata. Il termine è ripreso dall etnometodologia, che parla, a proposito delle attività dei membri, di embodied practices [...] embodied practice significa essenzialmente pratica situata, spazializzata e temporalizzata, fatta esistere da soggetti specifici, gli astrofisici Cocke o Disney, in un luogo specifico, e in condizioni specifiche (Semprini, 1995: 113).

El presente de la jornada laboral es lo que este nivel narrativo figurativiza para desarrollar la personalidad del signo Movistar. Para ello, en primer lugar, son escogidas unas personas famosas que adquirirán el rol de actantes sujetos en el discurso de la marca. Dichas personalidades son: Plácido Domingo, Marta Robles y Álex Crivillé quienes muestran y demuestran la funcionalidad y eficiencia de Movistar. Tres personajes diferentes pertenecientes a esferas distintas (música, periodismo y deporte), pero con una característica en común: la «elección» de tener Movistar. El discurso nos los muestra en el quehacer de su vida cotidiana destapando, pues, un espacio urbano laboral. A modo de ejemplo, analizamos el spot de Marta Robles. 
En él podemos observar a la periodista en el interior de su casa desayunando, después aparece vestida de forma elegante en el exterior de su edificio pidiendo un taxi. A continuación, la vemos en el interior del taxi para finalmente descubrirla en el interior de su trabajo: la radio. Este fragmento de la vida laboral de la locutora se caracteriza por la movilidad espacial en la dicotomía interior/exterior. Dicha dicotomía alterna el espacio del hogar con el espacio urbano en el que se superponen constantemente planos a cámara rápida.

¿Qué función tiene Movistar? ¿Cómo aparece representado? Mientras que todo fluye rapidísimo en la vida cotidiana laboral, la posesión del objeto permanece siempre en contacto con el sujeto, siendo la única constante del discurso.
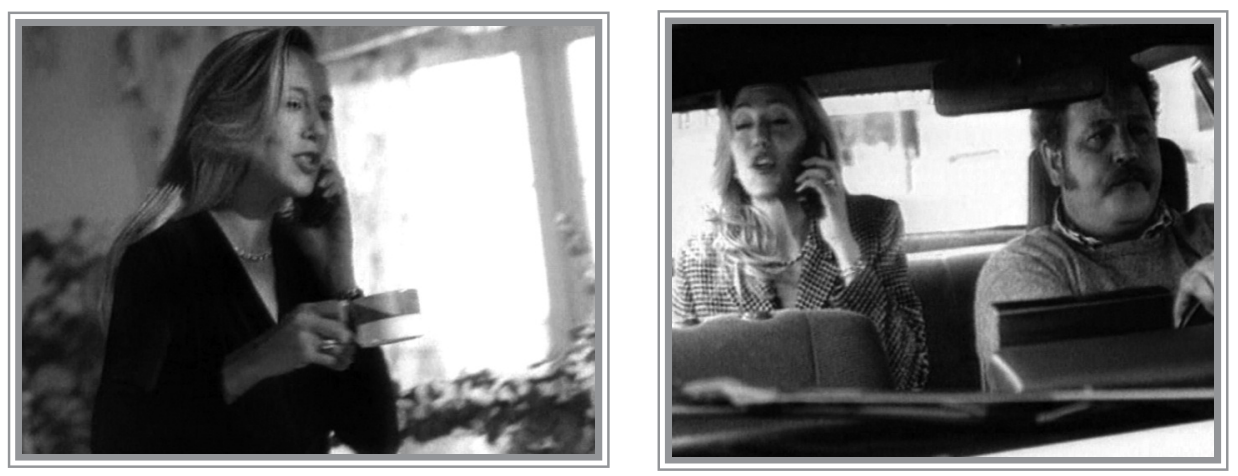

Fig. 2. Unión signo-usuario. Movistar (Telefónica, 1996)

Como vemos en este spot, la locutora tiene su brazo derecho elevado con el que sostiene el objeto que está utilizando desde la primera secuencia, a través del cual anuncia todo cuanto está sucediendo en ese preciso momento, mostrando de esta forma una de las grandes cualidades del signo en esta etapa: la ubicuidad, representada a través del concepto de dirección.

Pese a que todos los personajes tienen una meta, una dirección establecida, el discurso del relato focaliza la atención en el proceso, en el llegar, de modo que la duración presentista constituye otra de las características del signo. Estamos en un gerundio visual, todo está sucediendo ahora, en el mismo momento. Tanto es así que la primera secuencia de este spot es una muestra de ello, pues mientras que la locutora está «trabajando», retransmitiendo la 
noticia, en ese mismo momento su voz está siendo escuchada por un vecino quien se está afeitando a la vez que escucha la radio.

¿Qué cualidades adquieren los sujetos poseedores de Movistar? Experiencia, calidad y control eficaz. Estos tres conceptos son figurativizados en estas tres personalidades públicas las cuales están en acción, en movimiento rápido aunque «desestresante». Todos ellos representan la mejor profesionalidad cada uno en su campo. Con todo, la adversidad cotidiana también está presente.

\subsubsection{La adversidad controlada del viajero profesional. Reconocimiento del signo Movistar}

Tras la aparición de estas personalidades públicas, el discurso de la marca nos muestra segmentos de la jornada laboral de los ciudadanos anónimos. Analicemos el siguiente spot:

En él nos encontramos a una pareja leyendo el periódico en un bar al mismo tiempo que llaman por teléfono. A continuación observan una casa, la suya, que se acaban de comprar. En ese momento la felicidad de su expresión facial se torna en sorpresa desagradable dado el estado de abandono que presenta la casa.
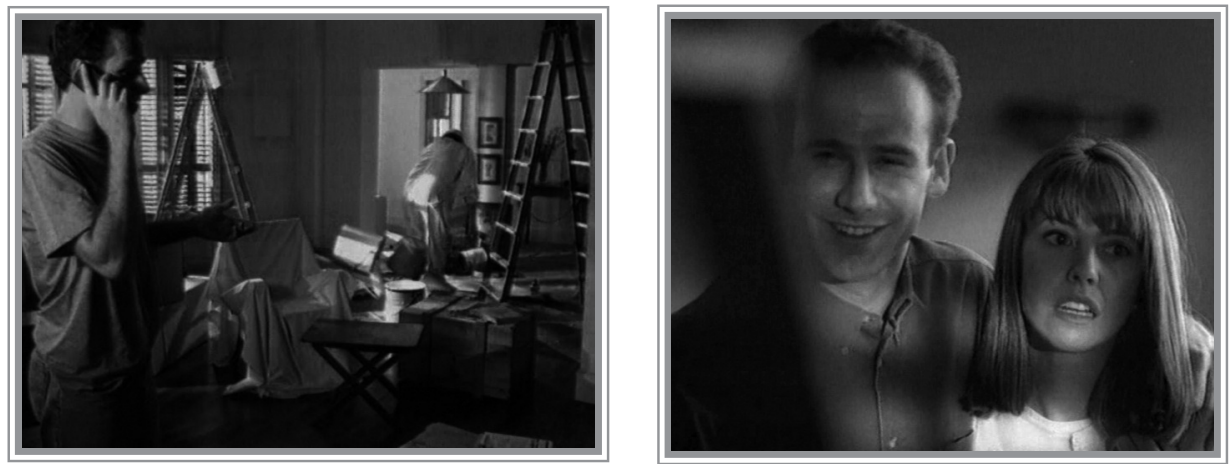

Fig. 3. Gestualidad ante la adversidad, Movistar (Telefónica, 1997)

Después, los personajes se separan para ocuparse cada uno de sus responsabilidades laborales con respecto a la casa, focalizando la atención en la chica que utiliza el teléfono desde este momento mientras se le ve comprando objetos decorativos y comida. El marido también utiliza el móvil mientras 
se ocupa de las responsabilidades del hogar desde su interior. Finalmente, se encuentran en su nuevo hogar mientras cenan y continúan hablando por el móvil.

La conjunción sujeto-objeto supone, además, el control ante la adversidad en la jornada laboral de estos viajeros anónimos, siendo esta característica otra de las cualidades del imaginario del signo. Dicha adversidad es representada tanto en el interior como en el exterior. Pero, ¿cómo se figurativiza el obstáculo?

Siguiendo con el último spot, en la Fig. 4 visualizamos el contraste oscuridad/luz (dicotomía constante tanto en Moviline como en Movistar). La casa está tímidamente iluminada por la claridad que entra del exterior a través de las ventanas. Cabe destacar la verticalidad de los «habitantes» de la casa hasta el momento: obsérvese la verticalidad de las ventanas, de los marcos de las puertas, del respaldo de la silla, de las tablas que componen una caja localizada en el suelo del inmueble. Dicho contraste lumínico se figurativiza al final del spot en un complemento decorativo cuya posesión se vuelve necesaria para los recién casados, pues supone el soporte lumínico en ese mismo momento. Estamos hablando de la figura de un perro dálmata caracterizado por su blancura salpicada de círculos negros en la figura vertical del cuerpo.

Como vemos, la verticalidad, lo circular, y la luz forman parte del imaginario identificativo de la marca Movistar para el usuario-consumidor.

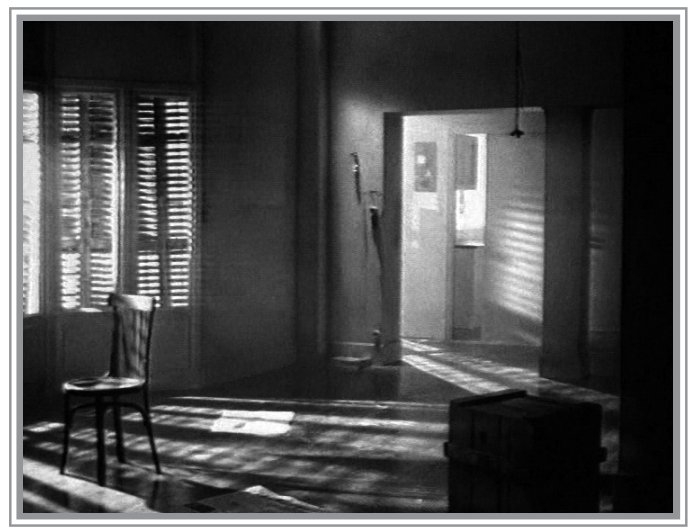

Fig. 4. Reconocimiento de la identidad del signo. Movistar (Telefónica, 1997)

Este spot figurativiza también una nueva adversidad anunciada lingüísticamente: la necesidad ante el comienzo de una nueva vida. 
El spot de Marta Robles nos muestra la adversidad en el exterior, la cual se centra en otra figurativización que ya encontramos en el relato de Moviline; nos estamos refiriendo al problema del tráfico, observado de manera reflectiva mediante el espejo de una ventana de un edificio por la locutora desde el taxi en el que se encuentra en ese mismo momento. Dicho atasco adopta la forma vertical triplicada y una tonalidad ambigua entre el blanco y el negro.

La identidad del imaginario está siempre haciéndose ver ante el usuarioconsumidor. A este respecto, hemos de destacar las palabras de Bassani, quien señala que cuando el consumidor ya ha asimilado la marca, un mínimo detalle le basta para identificarla:

Quando il consumatore ha assimilato la marca, gli è sufficiente un minimo segnale per risvegliarne con vividezza tutte le sensazioni. Le figure a fianco, per esempio, mostrano come l associazione di un colore, di una forma o di un carattere siano sufficienti a risvegliare l immaginario di marca (Bassani, 2002: 123).

Bassani (2002: 125), tras apuntar este pensamiento, establece un esquema en el que opone el hemisferio derecho al izquierdo, mostrando las funciones receptivas de ambos frente a un mensaje publicitario. Bassani señala que frente a un mensaje publicitario, la parte izquierda del cerebro analiza las palabras y los conceptos mientras que la parte derecha crea el sentido global.

El esquema de pensamiento es el siguiente: 


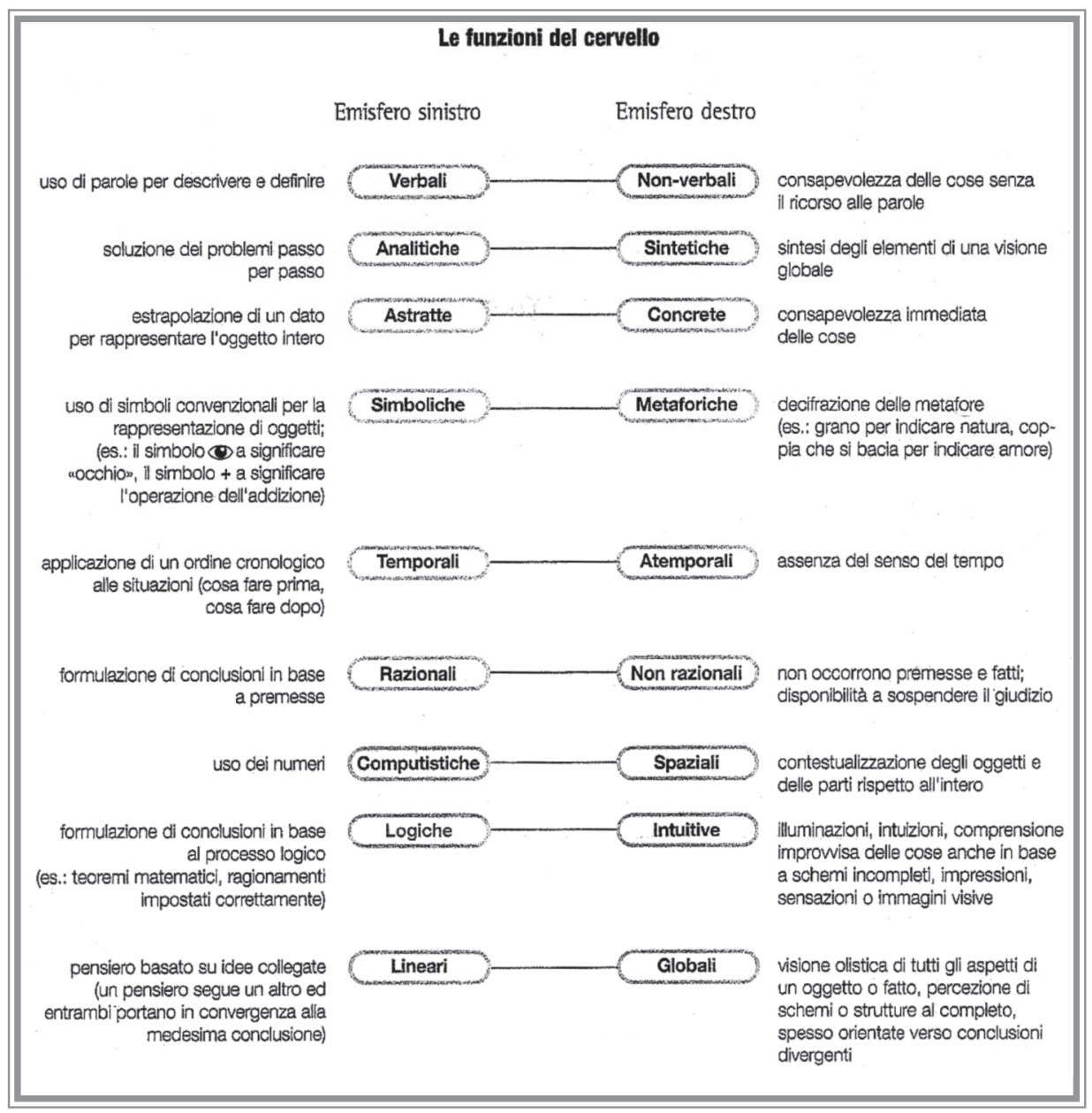

Tabla 1. Funciones receptivas de los hemisferios izquierdo y derecho ante el mensaje publicitario (Bassani, 2002: 125)

Detrás de las historias vividas por los sujetos-viajeros profesionales se halla implícito el concepto de novedad, de ahí el valor «utópico» desarrollado en el sujeto poseedor del objeto de valor, puesto que empezar de nuevo supone una nueva identidad en un nuevo espacio, en un nuevo tiempo.

Persona, tiempo, espacio, objeto; estamos ante un incipit, ante un comienzo nuevo revolucionador y desestructrador social. Todo ha cambiado. El discurso narrativo parte de ese comienzo para centrarse en el presente actual, para reflejar la acción, el proceso instantáneo. 
Dicha novedad, además de especificarse lingüísticamente se figurativiza de diversas formas en los spots analizados:

- Mediante el motivo de empezar una nueva vida, figurativizado en el vacío de la casa y en las primeras labores del comienzo hogareño.

- En la aceleración temporal del comienzo del día para detenerse en el presente cotidiano en el que transcurre la acción.

- En la especificación espacial como índice del nuevo tramo, del nuevo reto profesional.

\section{CONCLUSIONES}

A lo largo de este trabajo hemos podido comprobar cómo el producto Movistar es vaciado de sustancia e investido de nuevos valores significativos en su discurso a través de los cuales se muestra la relación entre el usuarioconsumidor y su objeto.

Pese a los cuatro valores propuestos por Floch, en este trabajo hemos analizado el utópico en dos spots de Movistar en España (Telefónica), pertenecientes a los años 1996 y 1997. Ambos muestran a diferentes sujetos que realizan su propia identidad adjuntos a su objeto de valor para controlar tanto su vida laboral como personal.

Movistar adquiere en estos años para el usuario-consumidor que lo lleva consigo como extensión de sí mismo, una personalidad de marca basada en la confianza, en la profesionalidad y en la solución ubicua ante cualquier obstáculo.

\section{REFERENCIAS BIBLIOGRÁFICAS}

BASSANI, M. y SBALCHIERO, S. (2002). Brand design. Costruire la personalità di marca vincente. Firenze: Alinea.

BASSO, P. L. (2003). Confini del cinema. Strategie estetiche e ricerca semiotica. Torino: Lindau.

BLACKETT, T. (1989). «The role of brand valuation in marketing strategy». Marketing and Research Today 17, 245-248.

DEL RÍO LANZA, A.; VÁZQUEZ CASIELLES, R. e IGLESIAS ARGÜELLES, V. (2002). «El valor de marca: perspectivas de análisis y criterios de estimación». Cuadernos de Gestión 2, 87-102. 
FLOCH, J. M. (2002). Semiotica, marketing e comunicazione. Dietro i segni, le strategie. Milano: Franco Angeli.

GREIMAS, A. J. (1966). Sémantique structurale. Paris: Larousse.

KLEIN, N. (2005). No Logo. El poder de las marcas. Barcelona: Paidós.

LUSCH, R.F. y HARVEY, M.P. (1994). «The case for an off -balance- sheet controller». Slogan Management Review 2, 101-105.

MARRONE, G. (1999). C era una volta il telefonino. Un indagine sociosemiotica. Roma: Meltemi.

PELLICER GARCÍA, L. (2010): «Análisis semiótico-lingüístico del discurso de la marca moderna (I). El caso Moviline en España». PLP Pensar la Publicidad III-2, 149-166.

PÉREZ YUSTE, A. (2002). «El proceso de implantación de la telefonía móvil en España». Antena de Telecomunicación 153, 26-31.

TAUBER, E.M. (1988). «Brand leverage: Strategy for growth in a cost-control world». Journal of Advertising Research 1, 26-30.

SEMPRINI, A. (1993). Marche e mondi possibili. Un approccio semiotico al marketing della marca. Milano: Tipomonza.

SEMPRINI, A. (1995). La marque. Paris: Presses Universitaires de France.

Recibido el 24 de enero de 2011.

Aceptado el 16 de septiembre de 2011. 